\title{
Rhodochrosite as Crystal Oscillator
}

\author{
Ricardo Gobato ${ }^{1 *}$, Marcia Regina Risso Gobato ${ }^{2}$ and Alireza Heidari ${ }^{3}$ \\ ${ }^{1}$ Laboratory of Biophysics and Molecular Modeling Genesis, State Secretariat of Education of Parana, Brazil \\ ${ }^{2}$ Green Land Landscaping and Gardening, Seedling Growth Laboratory, Brazil \\ ${ }^{3}$ Faculty of Chemistry, California South University, USA
}

*Corresponding author: Ricardo Gobato, Laboratory of Biophysics and Molecular Modeling Genesis, State Secretariat of Education of Parana, Brazil.

To Cite This Article: Ricardo Gobato. Rhodochrosite as Crystal Oscillator. Am J Biomed Sci \& Res. 2019 - 3(2). AJBSR.MS.ID.000659.

DOI: 10.34297/AJBSR.2019.03.000659

Received: May 03, 2019 | Published: June 06, 2019

\section{Introduction}

The electric charge that accumulates in certain solid materials, such as crystals, certain ceramics, and biological matter such as bone, DNA and various proteins) in response to applied mechanical stress, phenomenon called piezoelectricity.

A crystal oscillator is an electronic oscillator circuit that uses the mechanical resonance of a vibrating crystal of piezoelectric material to create an electrical signal with a precise frequency. The most common type of piezoelectric resonator used is the quartz crystal, so oscillator circuits incorporating them became known as crystal oscillators. Particularly one using a quartz crystal, works by distorting the crystal with an electric field, when voltage is applied to an electrode near or on the crystal. This property is known as electrostriction or inverse piezoelectricity. When the field is removed, the quartz - which oscillates in a precise frequency generates an electric field as it returns to its previous shape, and this can generate a voltage. The result is that a quartz crystal behaves like an RLC circuit, but with a much higher Q. Quartz crystals are manufactured for frequencies from a few tens of kilohertz to hundreds of megahertz. More than two billion crystals are manufactured annually. Most are used for consumer devices such as wristwatches, clocks, radios, computers, cell phones, signal generators and oscilloscopes.

But other crystals such as rhodochrosite also have piezoelectric properties. The rhodochrosite as crystal oscillator for being an alternative to those of quartz. The rhodochrosite (MnC03) shows complete solid solution with siderite (FeCO3), and it may contain substantial amounts of $\mathrm{Zn}, \mathrm{Mg}, \mathrm{CO}$ and $\mathrm{Ca}$. The Kutnohorite [CaMn(CO3)2] is a dolomite group mineral intermediary between rhodochrosite and calcite.

A molecular dynamic via molecular mechanics using the UFF (Universal Forces Field) may provide the vibrational frequencies of the fundamental structure of the crystal molecule.

In short, the goal of molecular mechanics is to predict the detailed structure and physical properties of molecules. Examples of physical properties that can be calculated include enthalpies of formation, entropies, dipole moments, and strain energies. Molecular mechanics calculates the energy of a molecule and then adjusts the energy through changes in bond lengths and angles to obtain the minimum energy structure.

$$
E_{s e}=E_{s t r}+E_{\text {bend }}+E_{\text {str-bend }}+E_{\text {oop }}+E_{\text {tor }}+E_{v d w}+E_{q q}
$$

The steric energy, bond stretching, bending, stretch-bend, out of plane, and torsion interactions are called bonded interactions because the atoms involved must be directly bonded or bonded to a common atom. The van der Waals and electrostatic (qq) interactions are between non-bonded atoms.

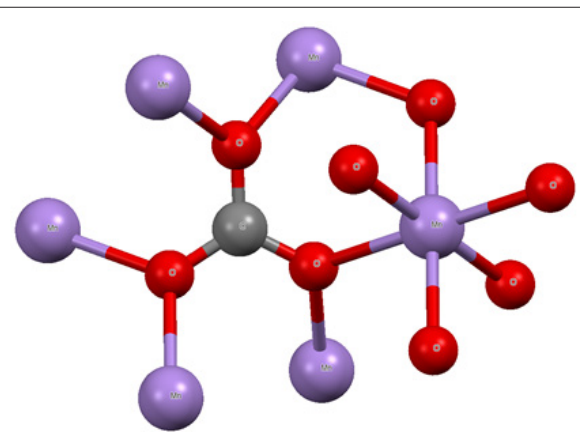

Figure 1: Cell structure of a rhodochrosite crystal. Represented in red color, Oxygen; in the dark gray color, the Carbon and in the purple color, the Manganese.

Calculations using Molecular Mechanics and methods ab initio in this direction, based on the unit molecular structure of the rhodochrosite crystal, (Figure 1), and experimental measurements could provide data from the pros and against the use of a crystal rhodochrosite oscillator, in an RLC circuit, replacing a crystal quartz oscillator (Figure 1).

Prospects for the insertion of nanoscale oscillating crystals into the bloodstream and their uptake by cancer cells, and their destruction by synchrotron radiation can be promising. 